\title{
Circulating Plasminogen Concentration at Admission in Patients with Coronavirus Disease 2019 (COVID-19)
}

\author{
Brandon Michael Henry, MD ${ }^{1, *}$ Stefanie W. Benoit, MD ${ }^{2,3, *}$ Jonathan Hoehn, BA \\ Giuseppe Lippi, MD ${ }^{5}$ Emmanuel J. Favaloro, PhD, FFSc(RCPA) ${ }^{6}$ Justin L. Benoit, MD
}

${ }^{1}$ Cardiac Intensive Care Unit, The Heart Institute, Cincinnati Children's Hospital Medical Center, Cincinnati, Ohio

2 Division of Nephrology and Hypertension, Cincinnati Children's Hospital Medical Center, Cincinnati, Ohio

${ }^{3}$ Department of Pediatrics, University of Cincinnati, College of Medicine, Ohio

${ }^{4}$ Department of Emergency Medicine, University of Cincinnati, Cincinnati, Ohio

${ }^{5}$ Section of Clinical Biochemistry, Department of Neuroscience, Biomedicine and Movement, University of Verona, Verona, Italy

${ }^{6}$ Department of Haematology, Sydney Centres for Thrombosis and Haemostasis, NSW Health Pathology, Institute of Clinical Pathology and Medical Research, Westmead Hospital, Westmead, New South Wales, Australia

Semin Thromb Hemost 2020;46:859-862.

The pathophysiology of coronavirus disease 2019 (COVID19), caused by severe acute respiratory syndrome coronavirus 2 (SARS-CoV-2), has puzzled the medical community, hampering the ability to generate efficient targeted therapies. ${ }^{1}$ Although SARS-CoV-2 is considered a primary respiratory pathogen, COVID-19 should be instead characterized as both a respiratory and systemic thrombotic disease. ${ }^{2}$ Conventionally termed COVID-19-associated coagulopathy (CAC), this hypercoagulable state is characterized by high risk of developing micro- and macrothromboemboli, impacting both arteries and veins. ${ }^{2}$ While initially affecting the pulmonary microvasculature, systemic involvement develops as the disease progresses, leading to thrombosis in distant organs and multiorgan dysfunction syndrome. ${ }^{2}$

The laboratory hallmarks of CAC include mildly prolonged prothrombin time, mild to moderate decrease of platelet count, and modestly elevated D-dimer values. ${ }^{3}$ Recently, Wright et al demonstrated a shutdown of fibrinolysis in patients with severe COVID-19 via high D-dimer and "total" failure of clot lysis at 30 minutes by thromboelastography (TEG). ${ }^{4}$ This was associated with significantly increased risk of thrombotic events and need for dialysis support. While acknowledging the inability of TEG to truly identify "total"

These authors share first authorship of this work.
Address for correspondence Brandon Michael Henry, MD, Cardiac Intensive Care Unit, The Heart Institute, Cincinnati Children's Hospital Medical Center, 3333 Burnet Avenue, Cincinnati, OH 45229 (e-mail: brandon.henry@cchmc.org).

failure of clot lysis due to technical limitations, multiple authors have hypothesized that suppression of fibrinolysis, in addition to hypercoagulability, substantially contributes to CAC, but the precise mechanisms remain unclear.,

Plasminogen is the precursor of plasmin, a protease that degrades the central components of the extracellular matrix, especially fibrin. Although plasminogen is classically considered inert, with fibrinolytic system regulated by a series of activators and inhibitors, evidence suggests that this enzyme may serve as a key regulator in many biologic processes, including fibrinolysis, tissue remodeling, angiogenesis, and wound healing. ${ }^{7,8}$

In addition to clot lysis, plasmin is important in the breakdown of hyaline membranes, a pathohistological hallmark of acute respiratory distress syndrome (ARDS), such as that caused by both SARS-CoV- 1 and SARS-CoV-2. ${ }^{7}$ These hyaline membranes, composed of a fibrin network intermingled with serum proteins and cellular debris, serve as barriers to gas exchange in the alveoli. In adults with ARDS, samples from bronchoalveolar lavage (BAL) have shown very high levels of plasminogen and active plasmin, as well as decreased activators and increased inhibitors of fibrinolysis, such as plasminogen activator inhibitor 1 (PAI-1). ${ }^{9,10}$ Given the ability for plasmin to break down hyaline membranes and promote wound healing, it has been hypothesized that

Copyright $\odot 2020$ by Thieme Medical Publishers, Inc., 333 Seventh Avenue, New York, NY 10001, USA.
DOI https://doi.org/ 10.1055/s-0040-1715454. ISSN 0094-6176. published online
September 3, 2020 Tel: +1(212) 760-0888. and Preventing Thrombosis in COVID-19

-Part I; Guest Editors: Emmanuel J. Favaloro, PhD, FFSc (RCPA), and Giuseppi Lippi, MD.
Issue Theme Maintaining Hemostasis 
supplementing fibrinolysis may improve clinical status in COVID-19. ${ }^{7}$ In a recent case series of 13 hospitalized patients with COVID-19, treatment with atomized inhaled freezedried plasminogen was effective to ameliorate lung lesions and reduce hypoxemia. ${ }^{7}$

Conversely, others have proposed that patients with elevated baseline plasminogen may be at increased risk of severe COVID-19, suggesting instead to target plasminogen with antiproteases. ${ }^{11}$ Similar to its established effect in SARS and other coronavirus diseases, plasmin in the airway cleaves the spike (S) protein of SARS-CoV-2, thus increasing its ability to bind to angiotensin-converting enzyme 2 (ACE2), the primary host receptor of the virus, fostering viral fusion with the plasma membrane and cell penetration. ${ }^{11}$ Moreover, plasmin cleaves epithelial sodium channel $(\mathrm{ENaC})$ subunits within the airway resulting in increased cellular sodium reabsorption, thus promoting pulmonary edema and airway dehydration in a manner similar to cystic fibrosis. ${ }^{11}$

To the best of our knowledge, no study to date has investigated plasminogen levels in patients with COVID-19. Given the potential for plasminogen to significantly contribute to COVID-19 pathophysiology, we report measurement of circulating plasminogen in patients with this pandemic infectious disease.

Adults with symptoms suggestive of COVID-19 who presented to the Emergency Department (ED) of the University of Cincinnati Medical Center (UCMC) were prospectively enrolled via an institutional review board-approved waiver of informed consent. All patients received a standard-of-care reverse transcription polymerase chain reaction (RT-PCR) test for COVID-19 via nasopharyngeal swab. RT-PCR-positive patients were diagnosed in the ED with COVID-19, while RTPCR-negative patients were classified for study purposes as sick controls. These controls were further evaluated by chart review and serology testing to exclude false-negative RT-PCR results. COVID-19 patients were stratified into subgroups based on ED disposition (hospitalized vs. discharged) or need for intensive care unit (ICU) admission (ICU vs. no ICU).

Blood samples were collected via routine blood draws for clinical indications in the ED. Within 3 hours of collection, samples were centrifuged at $2,000 \mathrm{~g}$ for 15 minutes at $4^{\circ} \mathrm{C}$ and subsequently frozen at $-80^{\circ} \mathrm{C}$ until analysis. Circulating (plasma) plasminogen was measured using a Behring Nephelometer II System (BN II; Siemens Medical Solutions USA, Inc.). Categorical values were compared using Fischer's exact test. Comparisons of plasma plasminogen concentrations and other continuous variables were performed using Mann-Whitney U-test, with $p$-value $<0.05$ considered statistically significant.

A total of 60 patients were enrolled in this investigation, 40 with RT-PCR-confirmed COVID-19 and 20 sick controls (comparison group). The characteristics of COVID-19 patients are presented in - Table 1. No difference was found between COVID-19 patients and sick controls with respect to sex $(p=0.781)$, age $(p=0.457)$, rate of hospitalization $(p=0.779)$, or need for ICU admission $(p=0.999)$.

All patients in both groups were within the 2.5th-97.5 percentile reference interval for plasminogen plasma levels
Table 1 Baseline characteristics of COVID-19 positive patients $(n=40)$

\begin{tabular}{|c|c|}
\hline Age $(y)$ : median (IQR) & $51.5(39.5-68.5)$ \\
\hline Sex (male): $n(\%)$ & $23(57.5 \%)$ \\
\hline \multicolumn{2}{|l|}{ Comorbidities } \\
\hline Coronary artery disease & $8(20.0 \%)$ \\
\hline Heart failure & $9(22.5 \%)$ \\
\hline Hypertension & $18(45.0 \%)$ \\
\hline Hyperlipidemia & $12(30.0 \%)$ \\
\hline Diabetes & $13(32.5 \%)$ \\
\hline $\begin{array}{l}\text { Chronic obstructive } \\
\text { pulmonary disease }\end{array}$ & $7(17.5 \%)$ \\
\hline Chronic kidney disease & $6(15.0 \%)$ \\
\hline Chronic liver disease & $6(12.5 \%)$ \\
\hline Cerebrovascular disease & $3(7.5 \%)$ \\
\hline Cancer & $2(5.0 \%)$ \\
\hline Obesity & $7(17.5 \%)$ \\
\hline Current smoker & $7(17.5 \%)$ \\
\hline Former smoker & $8(20.0 \%)$ \\
\hline \multicolumn{2}{|l|}{ Labs at admission } \\
\hline $\begin{array}{l}\text { White blood cells }\left(\times 10^{9}\right) \text { : } \\
\text { median }(\mathrm{IQR})\end{array}$ & $7.2(5.3-9.5)$ \\
\hline Platelets $\left(\times 10^{9}\right)$ : median (IQR) & $211(157.0-276.3)$ \\
\hline Fibrinogen $(\mathrm{g} / \mathrm{L})$ : median $(\mathrm{IQR})^{\mathrm{a}}$ & $6.6(5.6-7.2)$ \\
\hline $\begin{array}{l}\text { D-dimer }(\mu \mathrm{g} / \mathrm{mL} \text { FEU): } \\
\text { median }(\mathrm{IQR})^{\mathrm{a}}\end{array}$ & $1.2(0.68-1.8)$ \\
\hline Hospitalized from ED: $n(\%)$ & $26(65.0 \%)$ \\
\hline Required ICU admission: $n(\%)$ & $12(30.0 \%)$ \\
\hline
\end{tabular}

Abbreviations: COVID-19, coronavirus disease 2019; ED, emergency department; FEU, fibrinogen equivalent units; ICU, intensive care unit; $I Q R$, interquartile range.

${ }^{a}$ Data available for hospitalized patients only.

(0.06-0.25 g/L). No difference was found in plasminogen values between COVID-19 patients and sick controls (0.14 [IQR: 0.120.16 ] vs. 0.13 [IQR: $0.12-0.16$ ] g/L; $p=0.632$; - Fig. 1A). However, hospitalized COVID-19 patients had mildly lower plasminogen plasma concentration than COVID-19 patients discharged from the $\mathrm{ED}(0.13$ [IQR: $0.12-0.15]$ vs. 0.15 [IQR: $0.14-0.18$ ] g/L; $p=0.027$; - Fig. 1B). Similarly, COVID-19 patients who required ICU admission had mildly lower plasminogen plasma values than those discharged from the ED (0.12 [IQR: 0.11-0.14] vs. 0.14 [0.13-0.16] g/L; $p=0.048$; - Fig. 1C).

Taken together, the results of this investigation would suggest that significantly abnormal plasminogen values are not commonplace in patients with COVID-19. However, there are several factors to consider when interpreting these findings. Though the liver is the main site of plasminogen production, small amounts are also synthesized in a variety of other tissues. As such, we cannot exclude that the plasma concentration would be nonrepresentative of lung milieu, which will require measurement of BAL samples to delineate. ${ }^{8}$ 

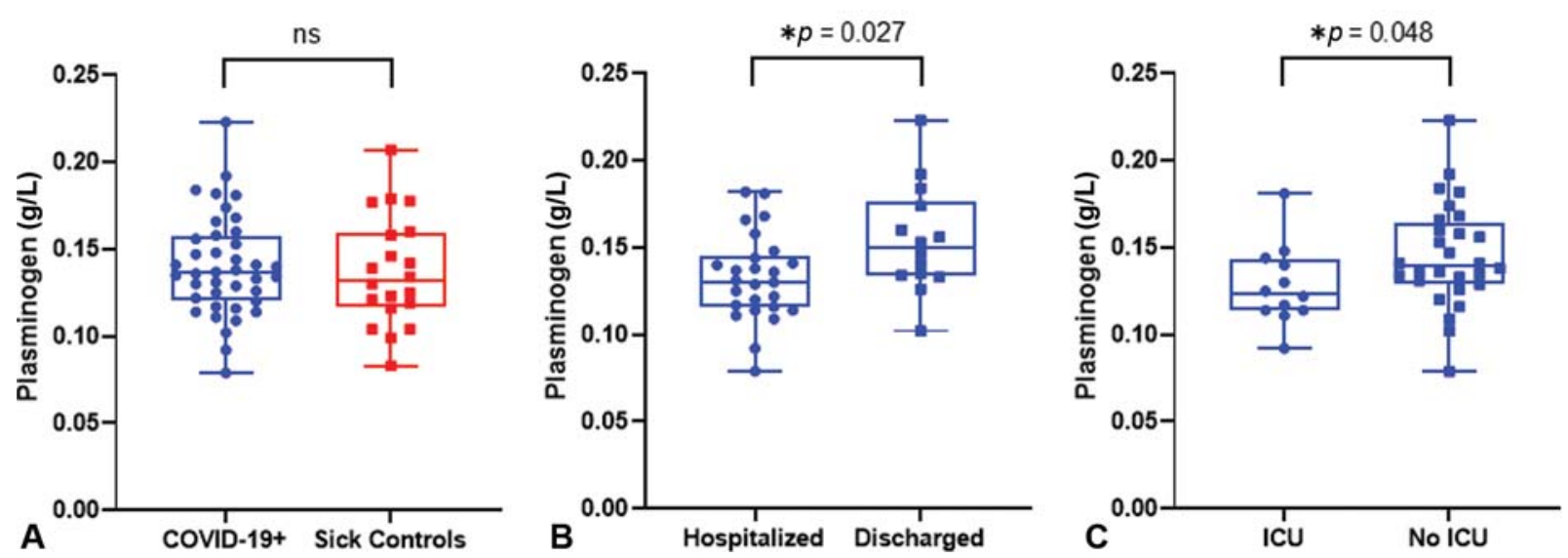

Fig. 1 Plasminogen concentration in COVID-19-positive patients versus COVID-19-negative sick controls (A), COVID-19-positive patients hospitalized versus discharged at initial presentation in emergency department (B), and in COVID-19-positive patients requiring ICU admission versus no ICU admission (C). COVID-19, coronavirus disease 2019; ns, nonsignificant.

We failed to observe higher levels of admission plasminogen in patients with COVID-19 compared with sick controls, thus limiting the hypothesized utility of antiproteases for treating COVID-19. Moreover, patients with elevated plasminogen at admission did not appear to progress to more severe disease. In fact, we observed a mild but significant trend of decreasing plasminogen concentration with increasing disease severity. Though no study has reported plasma plasminogen concentrations in patients with ARDS, our findings in the ICU group of COVID-19 patients are contradictory to those observed in BAL samples of ARDS, in which very high levels of plasminogen have been reported. ${ }^{9,10}$ We would expect that very high levels of local plasminogen production would have some circulatory spillover that could be detected in plasma, but this was not observed. Future studies should simultaneously measure BAL and plasma plasminogen and other fibrinolysis system components in patients with ARDS and/or COVID-19.

Although plasminogen in patients with COVID-19 is not significantly abnormal, the mild decrease observed in sicker COVID-19 patients does require pathophysiologic consideration for understanding the mechanism of CAC. The most likely explanation is that plasminogen is consumed by breaking down clots (as suggested by elevated D-dimer among hospitalized COVID-19 patients in this study), as well as breaking down hyaline membranes within alveoli. Importantly, high D-dimer values have been associated with poor COVID-19 outcomes. ${ }^{12}$ However, elevated concentration of D-dimer may also reflect excessive quantities of intravascular polymerized fibrin. ${ }^{4}$ Fibrin can serve as a cofactor for self-destruction, as fibrin enhances co-localization opportunities for plasminogen and tissue plasminogen activator (tPA). ${ }^{4,13}$ A second hypothesis may be that fibrinolysis is triggered by a viral activator, which causes plasminogen consumption and elevated tPA. ${ }^{4}$ Third, increased binding of plasminogen to receptors expressed on endothelium and inflammatory cells (monocytes and macrophages), which play a diverse role in the regulation of inflammation and wound healing, may also explain the observed declining trend with disease severity. ${ }^{14}$ Finally, elevated bradykinin due to viral-mediated inflammation may lead to increase tPA release from vascular endothelium, thus resulting in plasminogen consumption. ${ }^{15}$ Additionally, as ACE2 is involved in metabolism of bradykinin, attenuation of enzyme activity due to viral binding and internalization may in turn lead to increased bradykinin, further promoting tPA release. ${ }^{16,17}$

Given the trend toward lower plasminogen in more severe disease, it may be reasonable to attempt treatment with atomized inhaled plasminogen, as performed in the case series by Wu et al. ${ }^{7}$ However, plasminogen levels were not found at the level to meet a threshold of hypoplasminogenemia, nor at such low values to explain the low TEG clot lysis suggestive of fibrinolysis shutdown, as observed by Wright et al. ${ }^{4}$ Based on our results, it seems unlikely that low plasminogen is a major driver of CAC. However, we measured values at the time of initial $\mathrm{ED}$ presentation, and it is possible that an event later in disease course may result in rapid consumption, followed by inhibition of fibrinolysis. Serial plasminogen measurements will be necessary in future studies to delineate this potential mechanism. Nonetheless, given the plasma concentrations of plasminogen observed in this study, other causes of hypofibrinolysis should be evaluated in COVID-19. It has been hypothesized that inflammation triggers high levels of PAI-1, which may explain inhibition of fibrinolysis in the presence of adequate plasminogen. ${ }^{5}$ Thus, the use of tPA early in disease course may be a potential therapeutic strategy in patients with (or at higher risk of) severe disease. ${ }^{18} \mathrm{~A}$ case series by Wang et al of three patients with COVID-19 ARDS and respiratory failure found that tPA administration was associated with temporary improvement in respiratory status, as measured by partial pressure of oxygen:fraction of inspired oxygen (P:F) ratio, and similar clinical trials are ongoing. ${ }^{19}$

Many diseases may be associated with elevated baseline plasminogen levels, including hypertension, cardiovascular disease, cerebrovascular disease, diabetes, and chronic kidney disease. ${ }^{11}$ While these conditions foster an increased risk of severe COVID-19 illness, ${ }^{20-24}$ this study was not powered to compare plasminogen levels stratified by underlying 
comorbidities. Some patients included in our study had chronic liver disease, which, if severe, could potentially result in lower plasminogen levels along with that of other hepaticproduced proteins. Nonetheless, measured values for all patients in this study were within normal limits. Phipps et al reported in a large multicenter study that $6.4 \%$ of COVID19 patients develop severe acute liver injury (alanine transaminase five times the upper limit of normal). ${ }^{25}$ However, severe acute liver injury was not observed in any patient included in our cohort at the time of sample collection.

In conclusion, we observed that patients with COVID-19 present to the ED with normal levels of plasminogen, but those progressing to severe disease demonstrate mildly lower values suggestive of consumption for fibrinolysis activation. Future studies aimed at investigating the pathophysiology of CAC should include serial measurements of multiple components of the fibrinolytic system.

\section{Funding}

This study was funded by the University of Cincinnati College of Medicine Special Coronavirus (COVID-19) Research Pilot Grant Program.

\section{Conflict of Interest}

None.

\section{References}

1 Lippi G, Sanchis-Gomar F, Henry BM. Coronavirus disease 2019 (COVID-19): the portrait of a perfect storm. Ann Transl Med 2020; 8(07):497

2 Lippi G, Sanchis-Gomar F, Henry BM. COVID-19: unravelling the clinical progression of nature's virtually perfect biological weapon. Ann Transl Med 2020;8(11):693

3 Henry BM, de Oliveira MHS, Benoit S, Plebani M, Lippi G. Hematologic, biochemical and immune biomarker abnormalities associated with severe illness and mortality in coronavirus disease 2019 (COVID-19): a meta-analysis. Clin Chem Lab Med 2020;58 (07):1021-1028

4 Wright FL, Vogler TO, Moore EE, et al. Fibrinolysis shutdown correlation with thromboembolic events in severe COVID-19 infection. J Am Coll Surg 2020; Doi: 10.1016/j.jamcollsurg.2020.05.007 (epub ahead of print)

5 Henry BM, Vikse J, Benoit S, Favaloro EJ, Lippi G. Hyperinflammation and derangement of renin-angiotensin-aldosterone system in COVID-19: a novel hypothesis for clinically suspected hypercoagulopathy and microvascular immunothrombosis. Clin Chim Acta 2020;507:167-173

6 Kwaan HC. Coronavirus disease 2019: the role of the fibrinolytic system from transmission to organ injury and sequelae. Semin Thromb Hemost 2020; Doi: 10.1055/s-0040-1709996 (epub ahead of print)

7 Wu Y, Wang T, Guo C, et al. Plasminogen improves lung lesions and hypoxemia in patients with COVID-19. QJM 2020; Doi: 10.1093/qjmed/hcaa121 (epub ahead of print)

8 Aĭsina RB, Mukhametova LI. [Structure and functions of plasminogen/plasmin system]. Bioorg Khim 2014;40(06):642-657
9 Günther A, Mosavi P, Heinemann S, et al. Alveolar fibrin formation caused by enhanced procoagulant and depressed fibrinolytic capacities in severe pneumonia. Comparison with the acute respiratory distress syndrome. Am J Respir Crit Care Med 2000; 161(2, Pt 1):454-462

10 Idell S, James KK, Levin EG, et al. Local abnormalities in coagulation and fibrinolytic pathways predispose to alveolar fibrin deposition in the adult respiratory distress syndrome. J Clin Invest 1989;84(02):695-705

11 Ji H-L, Zhao R, Matalon S, Matthay MA. Elevated plasmin(ogen) as a common risk factor for COVID-19 susceptibility. Physiol Rev 2020;100(03):1065-1075

12 Lippi G, Favaloro EJ. D-dimer is associated with severity of coronavirus disease 2019: a pooled analysis. Thromb Haemost 2020;120(05):876-878

13 Collen D. Molecular mechanisms of fibrinolysis and their application to fibrin-specific thrombolytic therapy. J Cell Biochem 1987;33(02):77-86

14 Miles LA, Parmer RJ. Plasminogen receptors: the first quarter century. Semin Thromb Hemost 2013;39(04):329-337

15 Brown NJ, Gainer JV, Murphey LJ, Vaughan DE. Bradykinin stimulates tissue plasminogen activator release from human forearm vasculature through $\mathrm{B}(2)$ receptor-dependent, NO synthase-independent, and cyclooxygenase-independent pathway. Circulation 2000;102(18):2190-2196

16 de Maat S, de Mast Q, Danser AHJ, van de Veerdonk FL, Maas C. Impaired breakdown of bradykinin and its metabolites as a possible cause for pulmonary edema in COVID-19 infection. Semin Thromb Hemost 2020; Doi: 10.1055/s-0040-1712960 (epub ahead of print)

17 Sodhi CP, Wohlford-Lenane C, Yamaguchi Y, et al. Attenuation of pulmonary ACE2 activity impairs inactivation of des-Arg ${ }^{9}$ bradykinin/BKB1R axis and facilitates LPS-induced neutrophil infiltration. Am J Physiol Lung Cell Mol Physiol 2018;314(01):L17-L31

18 Bikdeli B, Madhavan MV, Gupta A, et al; Global COVID-19 Thrombosis Collaborative Group. Pharmacological agents targeting thromboinflammation in COVID-19: review and implications for future research. Thromb Haemost 2020;120(07):1004-1024

19 Wang J, Hajizadeh N, Moore EE, et al. Tissue plasminogen activator (tPA) treatment for COVID-19 associated acute respiratory distress syndrome (ARDS): a case series. J Thromb Haemost 2020;18 (07):1752-1755

20 Lippi G, Henry BM. Chronic obstructive pulmonary disease is associated with severe coronavirus disease 2019 (COVID-19). Respir Med 2020;167(00):105941

21 Henry BM, Lippi G. Chronic kidney disease is associated with severe coronavirus disease 2019 (COVID-19) infection. Int Urol Nephrol 2020;52(06):1193-1194

22 Lippi G, Wong J, Henry BM. Hypertension and its severity or mortality in coronavirus disease 2019 (COVID-19): a pooled analysis. Polish Archives Internal Medicine 2020;130(04):304-309

23 Aggarwal G, Cheruiyot I, Aggarwal S, et al. Association of cardiovascular disease with coronavirus disease 2019 (COVID-19) severity: a meta-analysis. Curr Probl Cardiol 2020;45(08):100617

24 Aggarwal G, Lippi G, Michael Henry B. Cerebrovascular disease is associated with an increased disease severity in patients with coronavirus disease 2019 (COVID-19): A pooled analysis of published literature. Int J Stroke 2020;15(04):385-389

25 Phipps MM, Barraza LH, LaSota ED, et al. Acute liver injury in COVID19: prevalence and association with clinical outcomes in a large US cohort. Hepatology 2020. Doi:10.1002/hep.31404 [epub ahead of print] 